NASA Technical Memorandum 105926

\title{
Friction and Wear of Plasma-Deposited Diamond Films
}

Kazuhisa Miyoshi

Lewis Research Center

Cleveland, Ohio

Richard L.C. Wu

Universal Energy Systems, Inc.

Dayton, Ohio

Alan Garscadden and Paul N. Barnes

Wright Laboratory

Wright-Patterson Air Force Base, Ohio

and

Howard E. Jackson

University of Cincinnati

Cincinnati, Ohio

Prepared for the

International Conference on Metallurgical Coatings and Thin Films sponsored by the American Vacuum Society

San Diego, California, April 19-23, 1993 
Friction and wear of plasma-deposited diamond films

\author{
Kazuhisa Miyoshi \\ National Aeronautics and Space Administration \\ Lewis Research Center \\ Cleveland, Ohio 44135 \\ Richard L.C. Wu \\ Universal Energy Systems, Inc. \\ Dayton, Ohio 45432
Alan Garscadden and Paul N. Barnes
Wright Laboratory
Wright-Patterson Air Force Base \\ Dayton, Ohio 45433. \\ and \\ Howard E. Jackson \\ Department of Physics \\ University of Cincinnati \\ Cincinnati, Ohio 45221
}

\begin{abstract}
Reciprocating sliding friction experiments in humid air and in dry nitrogen and unidirectional sliding friction experiments in ultrahigh vacuum were conducted with a natural diamond pin in contact with microwave-plasma-deposited diamond films. Diamond films with a surface roughness ( $\mathrm{R} \mathrm{rms}$ ) ranging from 15 to $160 \mathrm{~nm}$ were produced by microwave-plasmaassisted chemical vapor deposition. In humid air and in dry nitrogen, abrasion occurred when the diamond pin made grooves in the surfaces of diamond films, and thus the initial coefficients of friction increased with increasing initial surface roughness. The equilibrium coefficients of friction were independent of the initial surface roughness of the diamond films. In vacuum the friction for diamond films contacting a diamond pin arose primarily from adhesion between the sliding surfaces. In these cases, the initial and equilibrium coefficients of friction were independent of the initial surface roughness of the diamond films. The equilibrium coefficients of friction were 0.02 to 0.04 in humid air and in dry nitrogen, but 1.5 to 1.8 in vacuum. The wear factor of the diamond films depended on the initial surface roughness, regardless of environment; it increased with increasing initial surface roughness. The wear factors were considerably higher in vacuum than in humid air and in dry nitrogen.
\end{abstract}




\section{INTRODUCTION}

Because of recent innovations in the chemical vapor deposition process (CVD), synthetic diamond films can be grown economically on ceramics and metals for aerospace applications. Such applications range from diamond-coated cutting tools for composites, and wear resistant barriers for many moving mechanical assemblies, to electronics and radomes. ${ }^{1,2}$

The majority of diamond coatings produced at present are thin $(\sim 1 \mu \mathrm{m})$ films of polycrystalline diamond on ceramic or metal substrates. ${ }^{3,4}$ The surface roughnesses of these coatings are typically in the range of 10 to $1000 \mathrm{~nm}$ (center-line average), depending on the deposition conditions. Surface roughness is an important parameter that characterizes engineering surfaces used in tribological applications, and it generally affects the coefficient of friction.

The coefficients of friction for diamond films contacting diamond in air are in the range of 0.03 to $0.2 .^{3,4}$ The friction of diamond films in a vacuum has also been investigated..$^{5-7}$ However, only a few reports addressing the effect of surface roughness on the friction of diamond films have been published. ${ }^{3,4,8}$ And there have been no reports on the effect of surface roughness on the friction and wear behavior of diamond films under various environments, such as vacuum.

The objectives of the present study were to produce diamond films having a variety of grains, from fine to coarse, by controlling both the gas phase chemistry parameters (initial gas feed composition, pressure, flow rate, and plasma) and the surface chemistry parameters (surface treatment and surface temperature); to characterize the films by surface morphology, chemical composition, and grain size distribution; and further, to investigate the friction and wear behavior of these films in contact with a natural diamond pin in humid air, in dry nitrogen, and in vacuum, especially with regard to the effect of surface roughness on the friction and wear 
behavior. In earlier work, we demonstrated that it is possible to deposit fine grain (20 to $100 \mathrm{~nm}$ ) diamond films having surface roughness $\mathrm{R}$ rms $=15 \mathrm{~nm}$ by using a low pressure and low power microwave CVD technique. ${ }^{9}$

\section{EXPERIMENT}

\section{A. Diamond films}

The high-pressure microwave source plasma deposition system was used to deposit the diamond films. A detailed description of this apparatus is given elsewhere. ${ }^{9}$ Briefly, for this investigation diamond films were deposited on flat surfaces of (100) silicon, polycrystalline alpha-silicon carbide, and polycrystalline silicon nitride. The diamond nucleation density was enhanced by scratching the substrate with $0.5-\mu \mathrm{m}$ diamond paste. In order to produce the different surface morphologies, the deposition parameters controlling both the gas phase chemistry and the surface chemistry were systematically investigated. Detailed deposition conditions are listed in Table I.

A variety of techniques were used to characterize the as-deposited diamond films: scanning and transmission electron microscopy (SEM and TEM), to determine surface morphology and grain size measurements; Raman spectroscopy, to characterize diamond quality; $x$-ray diffraction, to determine the crystal orientation of diamond growth; surface profilometry, to measure the surface roughness by using a diamond stylus tip (12.5 $\mu \mathrm{m}$ in radius) that traversed at a load of $0.4 \mathrm{mN}(40 \mathrm{mg}$ ) over a scanning length of $2 \mathrm{~mm}$; and Rutherford backscattering (RBS) and proton recoil detection (PRD), to determine the composition of the films (the detector limit was on the order of 3 at.\%).

B. Friction and wear experiment

Two types of multipass sliding friction experiments were conducted with diamond films in contact with a natural, bulk diamond pin. The radius of curvature at the apex of the diamond pin used in the two kinds of experiments was $1.3 \mathrm{~mm}$. The (112) plane of the pin specimen was 
oriented such that it was nearly parallel to the sliding interface. All sliding was in the $<111>$ direction on the pin specimen. The bulk diamond pin was repolished with diamond powder ( $1 \mu \mathrm{m}$ in diameter) before each experiment (in dry nitrogen, in humid air, or in vacuum) and was ultrasonically rinsed in an ethanol bath. The two apparatuses and procedures used in this investigation are described in detail in references 10 and 11 .

Briefly, in the first type of multipass sliding friction experiment a pin-on-flat configuration was used. Reciprocating sliding friction experiments were conducted with a load of $1 \mathrm{~N}$ (mean Hertzian contact pressure, $2.2 \mathrm{GPa}$ ) in dry nitrogen at room temperature and in humid air at a relative humidity of approximately 40 percent [see Table II(a)]. Although the Hertzian contact pressure for a hard coating on a soft substrate (such as diamond film on a silicon or polycrystalline alpha-silicon carbide substrate) is theoretically smaller than for the solid hard materials (diamond-on-diamond), we calculated the mean Hertzian contact pressure (2.2 GPa) for the simple diamond-on-diamond contact, ignoring the potential role of the substrates. The real contact pressure should have been higher, because only selected tips of the asperities on the diamond surfaces contacted each other. ${ }^{7}$ Since the value of the elastic modulus of diamond films is the same as that for natural diamond, ${ }^{12}$ the latter $(1.05 \mathrm{TPa})$ was used to calculate the mean Hertzian contact pressure. ${ }^{13}$ The bulk diamond pin traveled back and forth (reciprocating motion), retracing its tracks on the diamond films. Sliding velocity was $86 \mathrm{~mm} \min ^{-1}$ over a track length of $3 \mathrm{~mm}$. Each experiment was conducted for $17.4 \mathrm{~h}$; thus, the pin passed over the track on the diamond film 30000 times during an experiment (a total sliding distance of $90 \mathrm{~m}$ ).

In the second type of multipass sliding experiment, a pin-on-disk configuration was used. Unidirectional, rotating sliding friction experiments were conducted with a load of $0.49 \mathrm{~N}$ (mean Hertzian contact pressure, $2.0 \mathrm{GPa})$ in an ultrahigh vacuum of $7 \times 10^{-7} \mathrm{~Pa}\left(5 \times 10^{-9} \mathrm{Torr}\right)$ at room temperature [see Table II(b)]. 
The friction force was continuously monitored during a friction experiment. Wear volumes of the diamond films were obtained by measuring the cross sectional area from stylus tracings across the wear tracks of at least four locations; then the average cross sectional area of the wear track was multiplied by the wear track length.

\section{RESULTS AND DISCUSSION}

\section{A. Characterization of diamond films}

\section{Surface morphology}

The diamond film surface morphology can be controlled by varying the gas phase chemistry parameters and surface chemistry parameters. Figure 1 shows the SEM photographs of fine-, medium-, and coarse-grain diamond films (experimental conditions are shown in Table I). The triangular crystalline facets typical of diamond are clearly shown in the medium and coarse diamond films, which have grain sizes estimated at 1100 and $3300 \mathrm{~nm}$, respectively. The sizes of the fine grain were determined from bright and dark field electron micrographs to be between 20 and $100 \mathrm{~nm}^{9}{ }^{9}$

The surface roughness of the diamond films was measured by a surface profilometer. The average surface roughness, which was calculated from a total of ten or more measurements, increased as the grain size increased, as shown in Fig. 2.

\section{Raman spectroscopy}

Typical Raman spectra of the fine- and medium-grain diamond films (Table I) are shown in

Fig. 3. Both spectra show one Raman band centered at $1333 \mathrm{~cm}^{-1}$ and one at around $1530 \mathrm{~cm}^{-1}$. The sharp peak at $1333 \mathrm{~cm}^{-1}$ is characteristic of the $\mathrm{sp}^{3}$ bonding of the diamond form of carbon in the film. The very broad peak centered around $1530 \mathrm{~cm}^{-1}$ is attributed to the $\mathrm{sp}^{2}$ bonding of the nondiamond forms of carbon (graphite and other carbon). ${ }^{14-16}$

More diamond was produced in the medium- and coarse-grain diamond films than in the fine-grain films, as is evident from the relative intensities of the diamond and nondiamond 
carbon Raman bands. However, the ratio of the intensities of the Raman response at $1333 \mathrm{~cm}^{-1}$ and $1530 \mathrm{~cm}^{-1}$ does not indicate the ratio of diamond to nondiamond carbon present in the film, since the Raman technique is approximately 50 times more sensitive to $\mathrm{sp}^{2}$-bonded (nondiamond) carbon than it is to $\mathrm{sp}^{3}$-bonded (diamond) carbon. ${ }^{15}$ Thus the peak at around $1530 \mathrm{~cm}^{-1}$ for each film represents a much smaller amount of nondiamond carbon in these films. ${ }^{16}$

\section{Carbon and hydrogen content of diamond films}

Rutherford backscattering was used to determine how much carbon was in the films and if there were any impurities in the films. Proton recoil detection was utilized to measure the hydrogen content. None of the films showed any element other than carbon and silicon. In the present study, the hydrogen content was estimated to be 2.5 at. $\%$ in the fine-grain films and less than 1 at.\% in the medium-grain films.

\section{4. $X$-ray diffraction analysis}

X-ray diffraction analysis (XRD) was used to determine the structure and crystal orientation of the diamond films listed in Table I. Typical XRD patterns for the fine- and medium-grain diamond films appear in Fig. 4. Peaks representing only diamond film and Si substrate appear in the XRD spectra. Diffraction peaks corresponding to (111), (220), (311), and (400), reflective of diamond, are clearly seen. The intensity ratio, $\mathrm{I}(220) / \mathrm{I}(111)$, was calculated from the XRD pattern for the fine- and medium-grain diamond films and found to be 1.3 and 0.04 , respectively. The powder diffraction pattern of diamond with random crystal orientation (ASTM 6-0675) gives $\mathrm{I}(220) / \mathrm{I}(111)=0.27$. Thus, most of the crystallites in the fine- and medium-grain diamond films are oriented with (110) and (111) planes, respectively, parallel to the surface. The well formed triangular facets observed in SEM photographs of medium- and coarse-grain diamond films confirm the (111) crystal orientation. 


\section{B. Friction and wear characteristics}

1. Humid air environment and dry nitrogen environment

In Fig. 5, the coefficients of friction obtained in typical experiments in humid air and in a dry nitrogen environment for fine-, medium-, and coarse-grain diamond films are plotted as functions of the number of repeated passes and the sliding distance. The coefficients of friction varied from point to point as the pin traveled back and forth. The values shown in Fig. 5 are the maximum values for each sliding pass. Overall, the friction behavior of fine-, medium-, and coarse-grain diamond films in contact with a bulk diamond pin in dry nitrogen was similar to their friction behavior in humid air: the coefficient of friction started relatively high (point A); it rapidly decreased after 60 to 200 passes (point B); and then it gradually decreased with an increasing number of passes, reaching an equilibrium value of between 0.03 and 0.04 (point C). The initial friction of the diamond films was 0.13 for fine-, 0.43 for medium-, and 0.52 for coarsegrain diamond films in humid air and 0.14 for fine-, 0.52 for medium-, and 0.69 for coarse-grain diamond films in dry nitrogen.

Thus the initial friction of the diamond films in humid air was generally lower than that in dry nitrogen; however, in both humid air and dry nitrogen it varied with the initial surface roughness, as can be seen in Fig. 6 where the initial coefficient of friction (i.e., point A in Fig. 5) is shown as a function of initial surface roughness of the diamond films. The initial surface roughness had little effect on the equilibrium coefficient of friction for the steady state condition, whether in humid air or in dry nitrogen [Fig. 6(b)].

In both the humid air and dry nitrogen environments, the diamond pin grooved the surfaces of diamond films. Stylus profilometer records typical of the wear tracks formed on the fine-, medium-, and coarse-grain diamond films are presented in Fig. 7. The lateral cross sectional area of the wear tracks indicates that the bulk diamond pin tends to dig into the surface of diamond films during sliding and produce a permanent groove. The groove surface is smoother 
than the original surface of the diamond films. Scanning electron microscopy analysis of the grooves revealed that wear occurred on the higher tips of the asperities of the diamond films and debris. Thus, the tips became dull, and the gaps between asperities were filled by debris.

Figure 8 presents the specific wear factor of the diamond films as a function of initial surface roughness. The specific wear factor obtained after 30000 passes was strongly dependent on the initial surface roughness of diamond films; it increased markedly with an increase in initial surface roughness.

\section{Vacuum environment}

Multipass unidirectional sliding friction experiments were conducted with the natural diamond pin contacting the diamond films in vacuum. A typical result for a run in vacuum is shown in Fig. 9. The coefficient of friction varied from point to point as the diamond pin moved around the track. Generally, the coefficient of friction increased with an increasing number of passes, reaching an equilibrium value after a certain number of passes, as seen in Fig. 9.

Figure 10 summarizes the initial and equilibrium coefficients of friction $\left(\mu_{\mathrm{I}}\right.$ and $\left.\mu_{\mathrm{F}}\right)$ for the diamond pin sliding on films in vacuum. The equilibrium coefficients of friction (1.5 to 1.8) obtained at steady state conditions were greater than the initial coefficients of friction (1.1 to 1.3), regardless of initial surface roughness of the diamond films. Further, these data indicate that in vacuum the initial surface roughness of diamond films has almost no effect on either the initial or equilibrium coefficient of friction.

The diamond pin grooved the surfaces of diamond films in vacuum. Typical profilometer records of the resulting wear tracks are presented in Fig. 11. The groove surface is generally smoother than the original surface of the diamond films. Further analysis of the grooves by scanning electron microscopy revealed that the tips of the diamond coating asperities were worn smooth, and the gaps between asperities were filled by debris. 
Figure 12 shows that the specific wear factor of the diamond films in vacuum was dependent on the initial surface roughness of the films; it generally increased with an increase in initial surface roughness.

C. Discussion on friction and wear of diamond films

\section{Friction in humid air and in dry nitrogen}

In humid air and in dry nitrogen, abrasion occurred and dominated the friction and wear behavior. The bulk diamond pin tended to dig into the surface of diamond films during sliding and produce a wear track (groove). When interactions between the diamond pin surface and the initially sharp tips of asperities on the diamond film surfaces were strong, the friction was high (point A in Fig. 5). Surface roughness of diamond films can have an appreciable influence on initial friction of diamond films: the greater the initial surface roughness, the higher the initial coefficient of friction. The frictional results in this report are consistent with the work of Casey and Wilks on single-crystal diamonds ${ }^{16}$ and the work of Hayward, Singer, and Seitzman on diamond coatings. ${ }^{4}$

As sliding continued and the pin passed repeatedly over the same track, the coefficient of friction was appreciably affected by the wear on the diamond films, that is, a blunting of the tips of asperities. When repeated sliding produced a smooth groove or a groove with blunted asperities on the surface of the diamond film (Fig. 7), the coefficient of friction was low, and the initial surface roughness effect became negligible; this showed that the equilibrium coefficient of friction was independent of the initial surface roughness of the diamond film [Fig. 6 (b)].

\section{Friction in vacuum}

In vacuum, as in humid air and in dry nitrogen, the bulk diamond pin dug into the surface of diamond films during sliding and produced a wear track (groove). However, the coefficient of friction increased with an increase in the number of passes (Fig. 9), just the opposite of that in humid air and in dry nitrogen. Further, the initial surface roughness of the diamond film had no 
effect on friction. These results lead us to ask, What factors determine the friction behavior? Which is more important for diamond surfaces in vacuum: abrasion or adhesion?

Removing some contaminant surface film from the contact area of diamond films results in a stronger interfacial adhesion between the diamond pin and diamond films and raises the coefficient of friction, as seen in Fig. 9. A contaminant surface film may be removed by repeatedly sliding the pin over the same track in vacuum. ${ }^{17}$

Our results are in agreement with other researchers' results for single-crystal diamond rubbing against diamond and for CVD diamond sliding against CVD diamond in vacuum. ${ }^{17,5}$ At a pressure of $9.3 \times 10^{-8} \mathrm{~Pa}$, Bowden and Hanwell ${ }^{17}$ observed an initial coefficient of friction of 0.1 for diamond on diamond; within several hundred passes, however, the coefficient of friction rose rapidly to 0.9 and remained constant. Dugger, Peebles, and Pope ${ }^{5}$ also found that the coefficient of friction increased to 0.47 when CVD diamond slid against itself in vacuum $\left(<6 \times 10^{-7} \mathrm{~Pa}\right)$. In both cases the increase in friction was attributed to cleaning the adsorbed contaminants from the surface by rubbing or sliding in vacuum at room temperature.

An opposite trend in friction behavior was observed in an investigation conducted on CVD diamond films in the relatively modest vacuum $\left(1.3 \times 10^{-3} \mathrm{~Pa}\right)$ of a scanning electron microscope. Gardos, Ravi, and Soriano ${ }^{6,7}$ found that the coefficient of friction began at 0.5 to 0.8 and dropped within several hundred passes. According to the authors, this was due to the removal of adsorbed oxygen and water vapor. Such an explanation for the decrease in initial friction, however, is not shared by Dugger, Peebles, and Pope, ${ }^{5}$ who see the decrease as more likely attributable to a reduction in surface roughness than to desorption of surface contaminants.

When sliding continues, the wear produces dull tips of the diamond grains and increases the contact area in the wear track, thereby causing an increase in friction. The increase in equilibrium friction that results from cleaning off the contaminant surface film by sliding and 
from increasing the contact area is greater than the corresponding decrease in friction that results from blunting the tips of surface asperities. This relationship is brought out clearly in Fig. 10; here the equilibrium coefficients of friction (1.5 to 1.8) are greater than the initial coefficients of friction (1.1 to 1.3 ) regardless of initial surface roughness of the diamond films. In vacuum, therefore, the friction arises primarily from adhesion between the sliding surfaces of the diamond pin and diamond films.

Gardos, Ravi, and Soriano ${ }^{6,7}$ found that the coefficients of friction for CVD diamond on CVD diamond in vacuum $\left(1.3 \times 10^{-3} \mathrm{~Pa}\right)$ sometimes increased to as high as 0.8 as the temperature increased. They attributed this high friction to the presence of dangling bonds on the surfaces of the diamond coatings. Dugger, Peebles, and Pope attributed the high "clean state" value of the coefficient of friction (as high as about 0.5 ) to the adhesive interaction of CVD diamond surfaces. ${ }^{5}$ Although we do not know the nature of the diamond-diamond bond, under the vacuum conditions of our experiment, it was adhesion between the sliding surfaces of the diamond pin and diamond films that played a significant role in the friction process; the surface roughness of the diamond films did not have much influence on the friction of diamond films in vacuum.

\section{Wear factor}

The generally accepted mechanism of wear for diamond is that of small fragments chipping off the surface. ${ }^{3}$ This is in agreement with our SEM observations of the diamond films and with our finding that the wear factor is very dependent on the initial surface roughness of diamond films, regardless of environment.

The wear factors of the diamond films in humid air and in dry nitrogen are comparable to the wear factors of single-crystal diamonds and diamond films. ${ }^{3,18,19}$ However, the wear factors of the diamond films in vacuum are considerably higher than those of diamond films in humid 
air or in dry nitrogen. Obviously, under these vacuum conditions, adhesion between the sliding surfaces of the diamond pin and diamond film plays an important role in the wear process.

\section{CONCLUDING REMARKS}

In our studies of the growth of diamond films and their physical characterization, we found that the surface morphology of diamond films can be controlled by varying both the gas phase chemistry parameters and the surface chemistry parameters during microwave chemical vapor deposition. The surface roughness ( $\mathrm{R} \mathrm{rms}$ ) and grain size of the diamond films in the present work ranged from 15 to $160 \mathrm{~nm}$ and 20 to $3300 \mathrm{~nm}$, respectively.

Although the Raman spectra demonstrated that all of the diamond films were of high quality, the fine-grain diamond films differed in a number of ways. They contained a relatively high concentration of nondiamond carbon, compared to the coarse- and medium-grain diamond films. In addition, the proton recoil detection data showed the fine-grain diamond films contained more hydrogen than did the coarse- and medium-grain diamond films. X-ray diffraction data also demonstrated that the crystallites in the fine-grain diamond film are oriented along (110), whereas those of the coarse- and medium-grain films are oriented along the (111) plane.

We found that the tribological characteristics of diamond films varied with the environment. The equilibrium coefficients of friction for diamond films were 0.02 to 0.04 in humid air and in dry nitrogen, but 1.5 to 1.8 in vacuum. Consequently, the wear factors of the diamond films were considerably higher in vacuum than in humid air and in dry nitrogen.

In humid air and in dry nitrogen, abrasion occurred when the diamond pin grooved the surfaces of diamond films. The initial coefficients of friction, therefore, increased with increasing initial surface roughness and were much higher than the equilibrium coefficients of friction. The equilibrium coefficients of friction were independent of the initial surface roughness of diamond films. 
In vacuum, the friction for diamond films contacting a diamond pin arose primarily from adhesion between the sliding surfaces. Both the initial and equilibrium coefficients of friction were independent of the initial surface roughness of diamond films. Further, the initial coefficients of friction were lower than the equilibrium coefficients of friction, in contrast to the measurements performed in humid air and in dry nitrogen.

The wear factor of the diamond films depended on the initial surface roughness of the diamond films, whether in humid air, in nitrogen, or in vacuum, and it generally increased with increasing initial surface roughness.

\section{ACKNOWLEDGMENTS}

This research activity was funded by an SDIO Contract (Universal Energy Systems, Inc.), sponsored by the Aero Propulsion and Power Directorate, Wright Laboratory, Wright Patterson Air Force Base, Ohio. The authors are grateful to Dr. A.K. Sarkar of the University of Dayton Research Institute for x-ray diffraction analyses. 


\section{REFERENCES}

${ }^{1}$ J.C. Angus and C.C. Hayman, Science 241, 913 (1988).

${ }^{2}$ L. Kempfer, Mater. Eng. 108, 28 (1991).

${ }^{3}$ I.P. Hayward, Surf. Coat. Technol. 49, 554 (1991).

${ }^{4}$ I.P. Hayward and I.L. Singer, Second International Conference Proceedings (Materials Research Society, Pittsburgh, PA, 1991), p. 785.

${ }^{5}$ M.T. Dugger, D.E. Peebles, and L.E. Pope, Surface Science Investigations in Tribology, edited by Y.-W. Chung, A.M. Homola, and G.B. Street (American Chemical Society, Washington, DC, 1992), p. 72.

${ }^{6}$ M.N. Gardos and K.V. Ravi, Electrochem. Soc. Proc. 89, 475 (1989).

${ }^{7}$ M.N. Gardos and B.L. Soriano, J. Mater. Res. 5, 2599 (1990).

${ }^{8}$ M. Kohzaki, K. Higuchi, S. Noda, and K. Uchida, J. Mater. Res. 7, 1769 (1992).

${ }^{9}$ R.L.C. Wu, A.K. Rai, A. Garscadden, P. Kee, H.D. Desai, and K. Miyoshi, J. Appl. Phys. 72, 110 (1992).

${ }^{10} \mathrm{~K}$. Miyoshi, Advances in Information Storage Systems (American Society of Mechanical Engineers, New York, 1991), Vol. 3, p. 147.

${ }^{11}$ K. Miyoshi, F.S. Honecy, P.B. Abel, S.V. Pepper, T. Spalvins, and D.R. Wheller, NASA TM-104478 (1992).

${ }^{12}$ C.J. McHargue, Proceedings of the First International Conference on the Applications of Diamond Films and Related Materials, edited by Y. Tzeng, M. Yoshikawa, M. Murakawa, and A. Feldman (Elsevier, New York, 1991), p. 113.

${ }^{13}$ S. Bhagavantam and J. Bhimasenacher, Proc. R. Soc. London (A) 187A, 381 (1946).

${ }^{14}$ J.J. Cheng, T.D. Mautei, R. Vuppulahadium, and H.E. Jackson, J. Appl. Phys. 71, 2918 (1992).

${ }^{15}$ K. Kobashi, K. Nishimura, V. Kawate, and T. Horiuchi, Phys. Rev. 38B, 4067 (1988). 
${ }^{16}$ N. Wada and S.A. Solin, Phys. B\&C 105B, 353 (1981).

${ }^{17}$ F.P. Bowden and A.E. Hanwell, Proc. R. Soc. London (A) 295A, 233 (1966).

${ }^{18}$ D. Crompton, W. Hirst, and M.H. Howse, Proc. R. Soc. London (A) 333A, 435 (1973).

${ }^{19}$ K. Miyoshi, R.L.C. Wu, and A. Garscadden, Surf. Coat. Technol. 54/55, 428 (1992). 
TABLE I. Deposition conditions for diamond films of various grain sizes.

\begin{tabular}{|c|c|c|c|c|c|c|c|c|c|c|}
\hline \multirow[t]{2}{*}{ Substrate ${ }^{a}$} & \multicolumn{3}{|c|}{$\begin{array}{l}\text { Gaseous } \\
\text { flow rate } \\
\left(\mathrm{cm}^{3} / \mathrm{min}\right)\end{array}$} & \multirow[t]{2}{*}{$\begin{array}{c}\text { Pressure } \\
\text { (Torr) }\end{array}$} & \multirow{2}{*}{$\begin{array}{l}\text { Micro- } \\
\text { wave } \\
\text { power } \\
(W)\end{array}$} & \multirow[t]{2}{*}{$\begin{array}{l}\text { Deposition } \\
\text { temperature } \\
\left({ }^{\circ} \mathrm{C}\right)\end{array}$} & \multirow[t]{2}{*}{$\begin{array}{c}\text { Deposition } \\
\text { time } \\
\text { (h) }\end{array}$} & \multirow[t]{2}{*}{$\begin{array}{c}\text { Thick- } \\
\text { ness } \\
\text { (nm) }\end{array}$} & \multirow[t]{2}{*}{$\begin{array}{l}\text { Grain } \\
\text { size } \\
(\mathrm{nm})\end{array}$} & \multirow{2}{*}{$\begin{array}{c}\text { Surface } \\
\text { rough- } \\
\text { ness } \\
\mathrm{R} \text { rms } \\
\text { (nm) }\end{array}$} \\
\hline & $\mathrm{CH}_{4}$ & $\mathrm{H}_{2}$ & $\mathrm{O}_{2}$ & & & & & & & \\
\hline $\operatorname{Si}(100)$ & 4 & 395 & 1 & 5 & 500 & $860 \pm 20$ & 10.5 & 1000 & $20-100$ & 15 \\
\hline $\operatorname{Si}(100)$ & 3.5 & 500 & 0 & 40 & 1000 & $1015 \pm 50$ & 14 & 4200 & 1100 & 63 \\
\hline$\alpha-\mathrm{SiC}$ & 3.5 & 500 & 0 & 40 & 1000 & $1015 \pm 50$ & 14 & 5000 & 3300 & 160 \\
\hline$\alpha-\mathrm{SiC}$ & 3.5 & 500 & 0 & 40 & 1000 & $965 \pm 50$ & 22 & 8000 & 1500 & 92 \\
\hline$\alpha-\mathrm{SiC}$ & 4 & 395 & 1 & 5 & 500 & $860 \pm 20$ & 21 & 1000 & $22-100$ & 50 \\
\hline $\mathrm{Si}_{3} \mathrm{~N}_{4}$ & 3.5 & 500 & 0 & 40 & 1000 & $965 \pm 50$ & 22 & 7000 & 1000 & 52 \\
\hline $\mathrm{Si}_{3} \mathrm{~N}_{4}$ & 4 & 395 & 1 & 5 & 500 & $860 \pm 200$ & 21 & 800 & $22-100$ & 35 \\
\hline
\end{tabular}

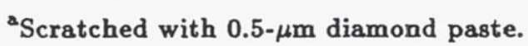

TABLE II. Conditions of friction and wear experiment.

(a) Dry nitrogen and humid air.

\begin{tabular}{|c|}
\hline .. Reciprocating \\
\hline 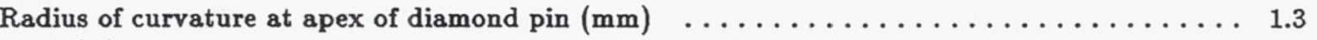 \\
\hline 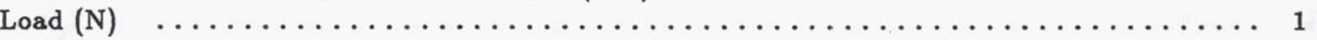 \\
\hline Initial average Hertzian contact pressure $(\mathrm{GPa}) \quad \ldots \ldots \ldots \ldots \ldots \ldots \ldots \ldots \ldots \ldots \ldots \ldots \ldots \ldots \ldots \ldots$ \\
\hline 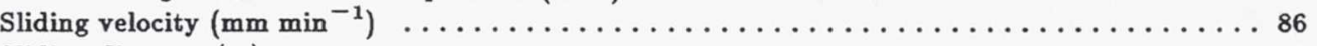 \\
\hline 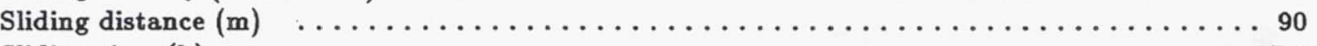 \\
\hline Sliding time $(\mathrm{h}) \ldots \ldots \ldots \ldots \ldots$ \\
\hline$\ldots$ Humid air at $\sim 40$-percent relative humidity \\
\hline 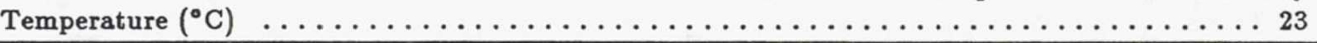 \\
\hline
\end{tabular}

(b) Vacuum.

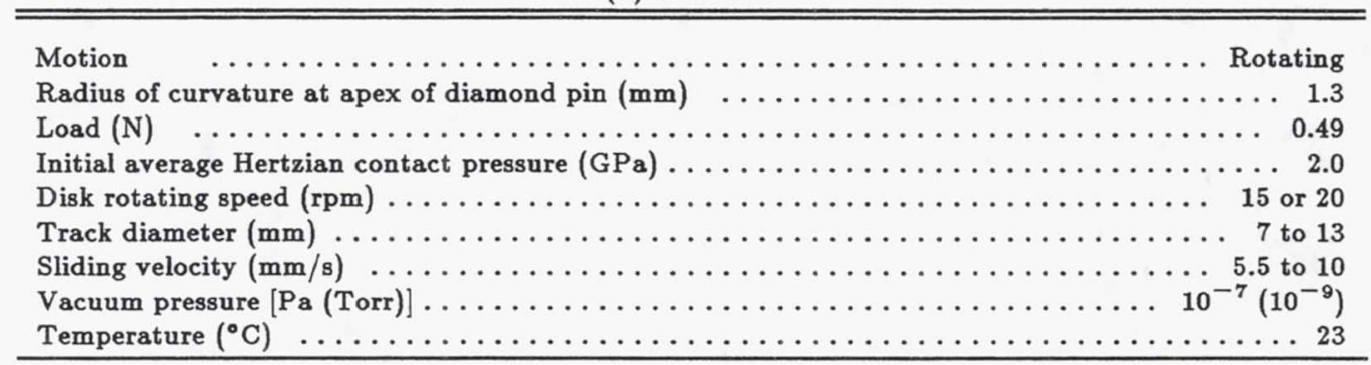




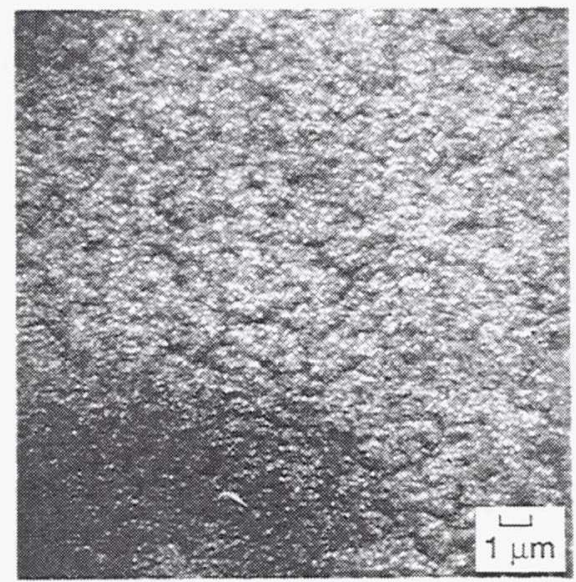

(a) Fine-grain (20 to $100 \mathrm{~nm}$ ) diamond film on (100) Si substrate; surface roughness $\mathrm{R}$ rms = $15 \mathrm{~nm}$.

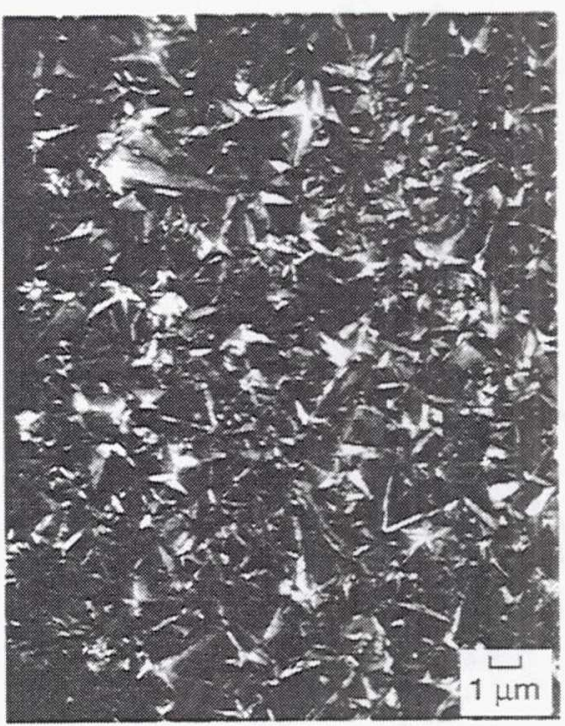

(b) Medium-grain (1100 nm) diamond film on (100) Si substrate; surface roughness $R \mathrm{rms}=$ $63 \mathrm{~nm}$

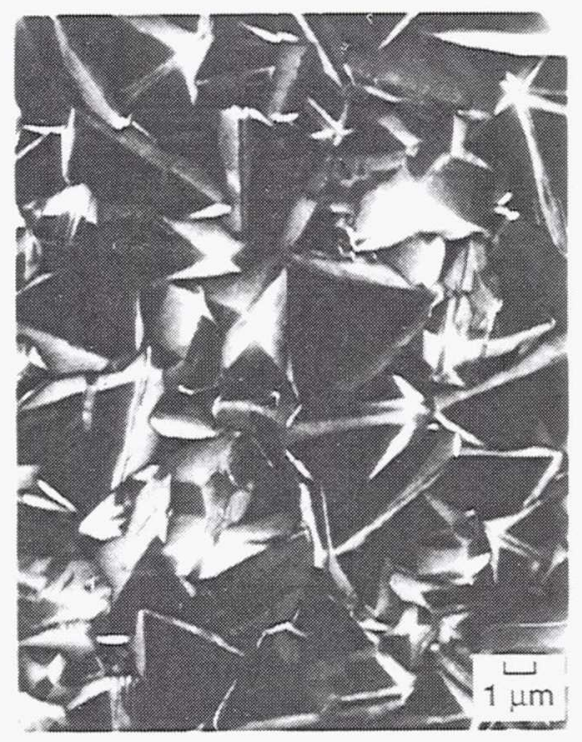

(c) Coarse-grain $(3300 \mathrm{~nm})$ diamond film on $\alpha-\mathrm{SiC}$ substrate; surface roughness $\mathrm{R} \mathrm{rms}=$ $160 \mathrm{~nm}$.

Figure 1. - Scanning electron micrographs of diamond films. 


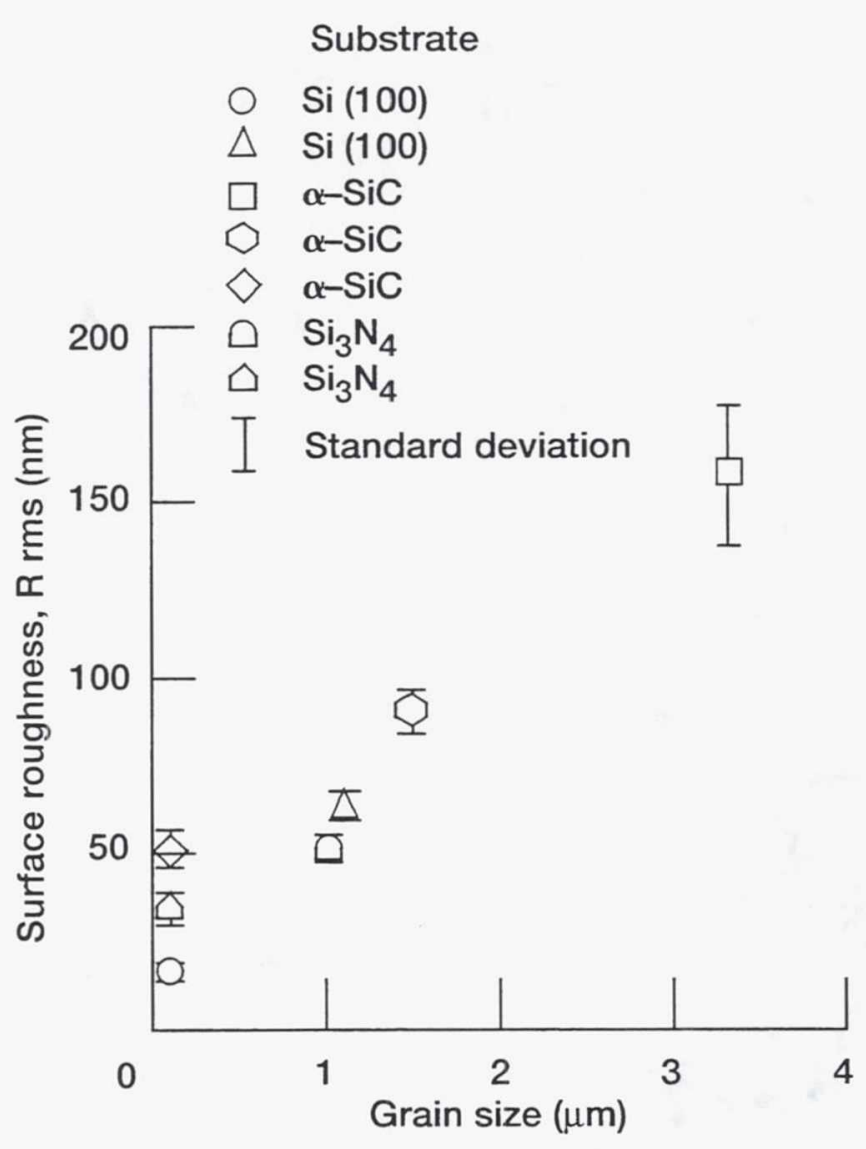

Figure 2. -Surface roughness as function of grain size for diamond films.

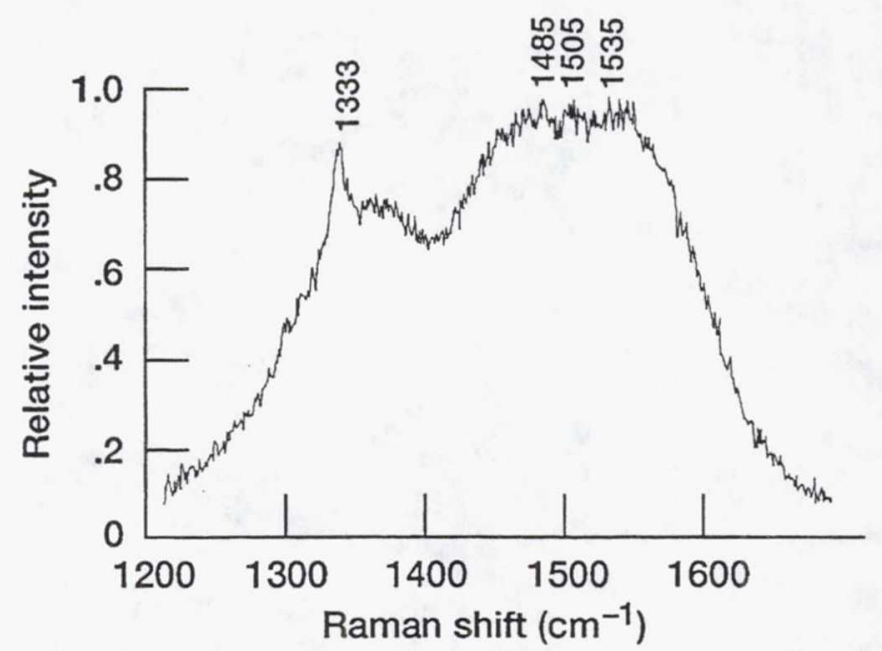

(a) Fine-grain (20 to $100 \mathrm{~nm}$ ) diamond film on (100) Si substrate.

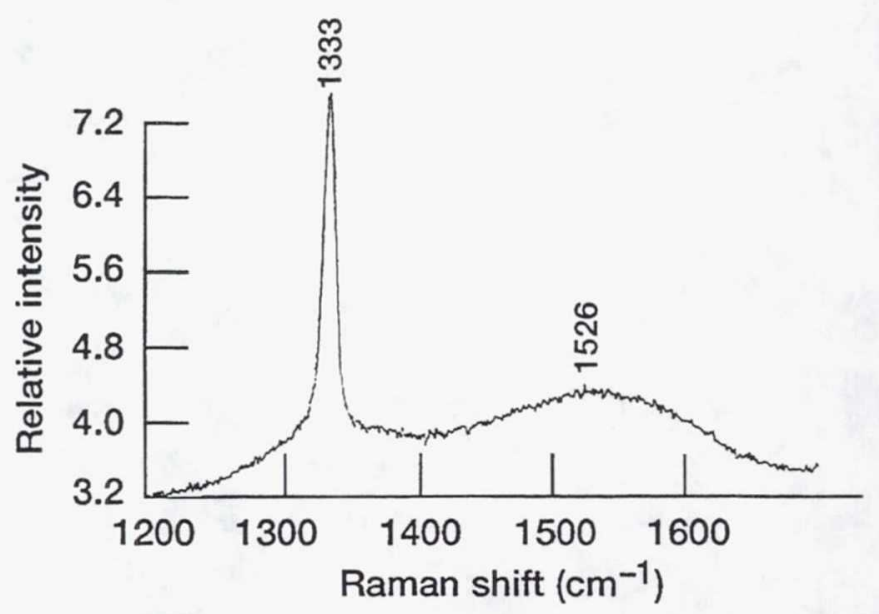

(b) Medium-grain $(1100 \mathrm{~nm})$ diamond film on (100) Si substrate.

Figure 3. - Raman spectra of diamond films. 


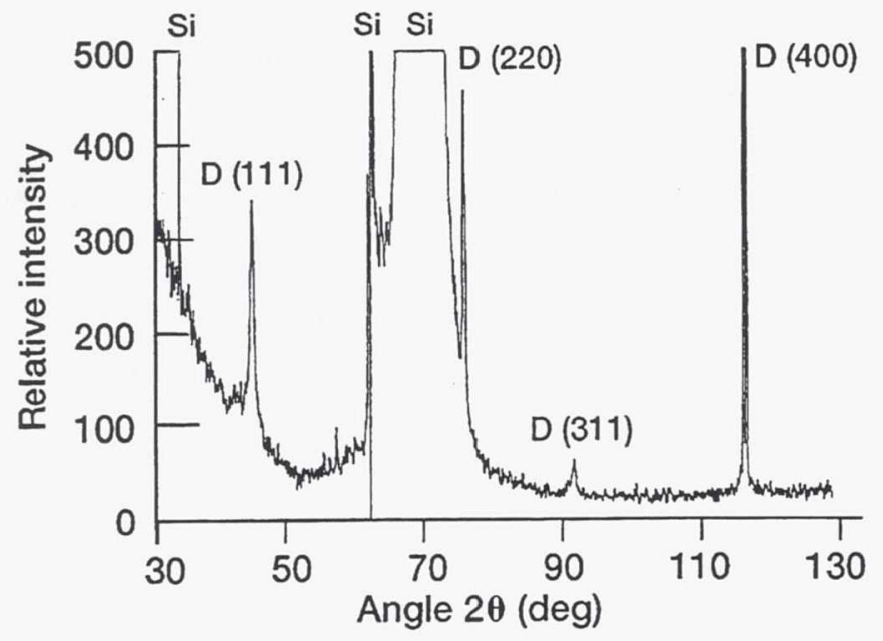

(a) Fine-grain (20 to $100 \mathrm{~nm}$ ) diamond film on (100) Si substrate.

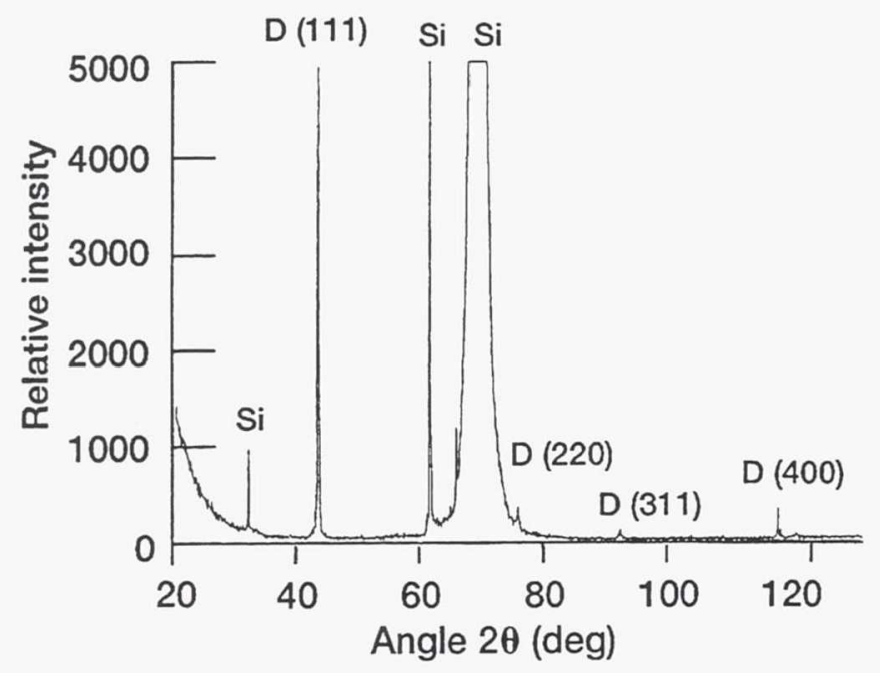

(b) Medium-grain $(1100 \mathrm{~nm})$ diamond film on (100) Si substrate.

Figure 4. - X-ray diffraction patterns of diamond films.

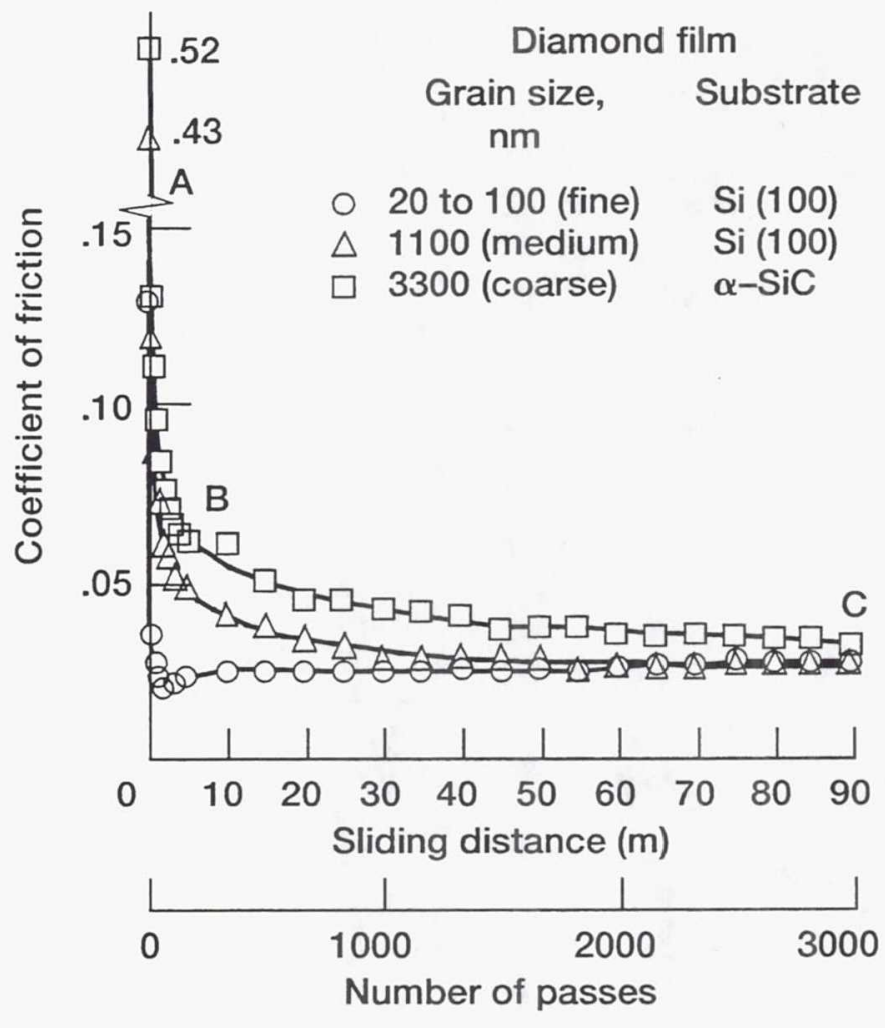

(a) Humid air at approximately 40-percent relative humidity.

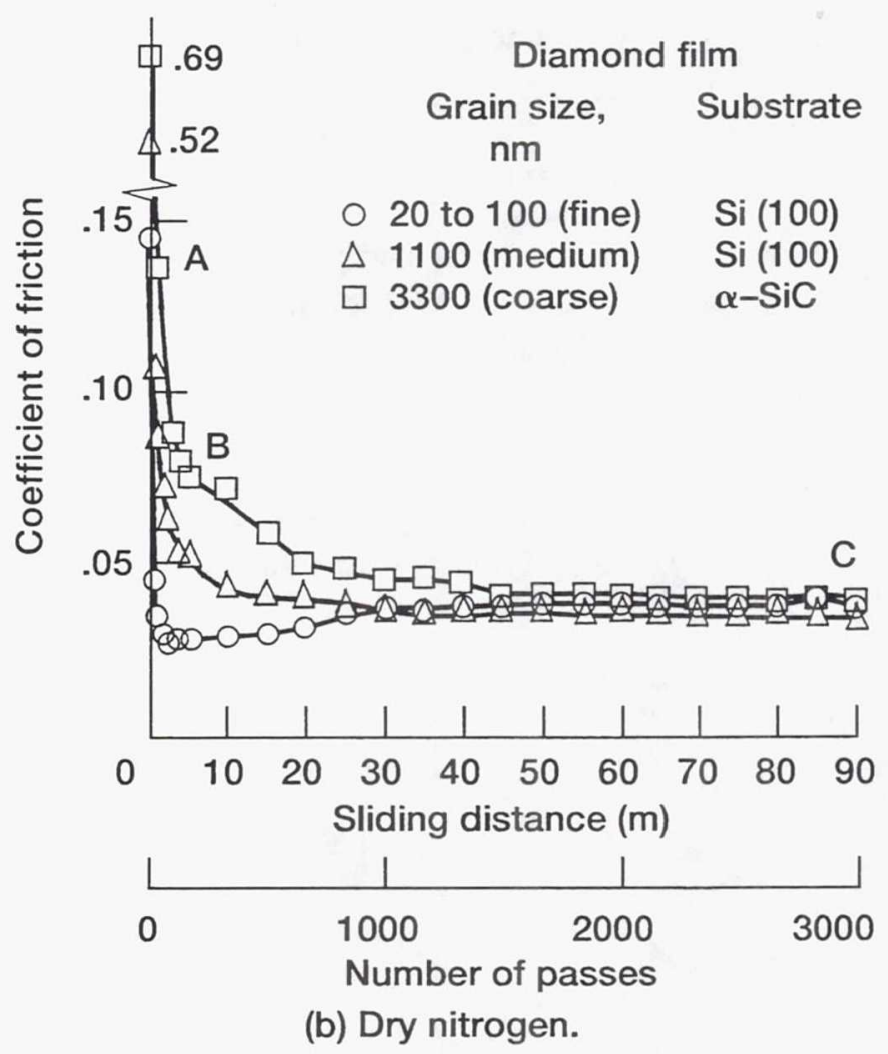

Figure 5. - Coefficient of friction as function of number of passes of bulk diamond pin in contact with fine-, medium-, and coarse-grain diamond films in humid air and in dry nitrogen. 


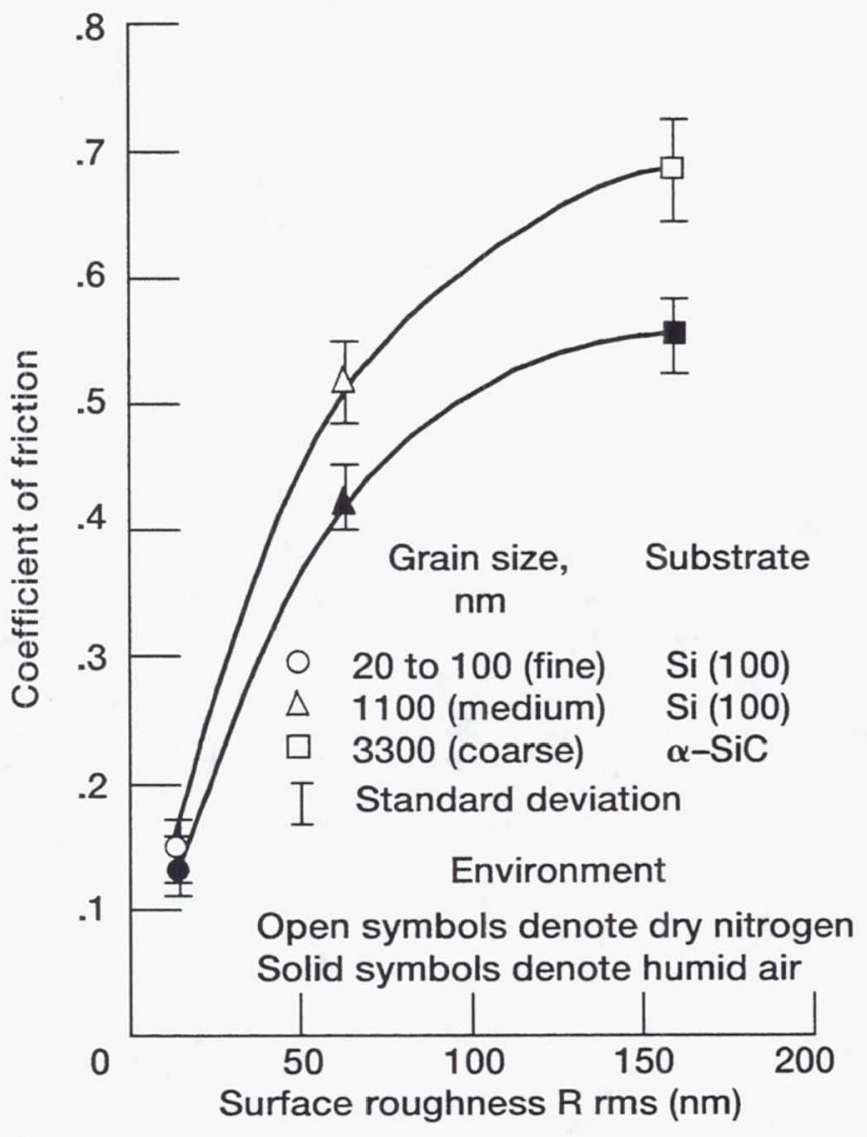

(a) Initial coefficients of friction.

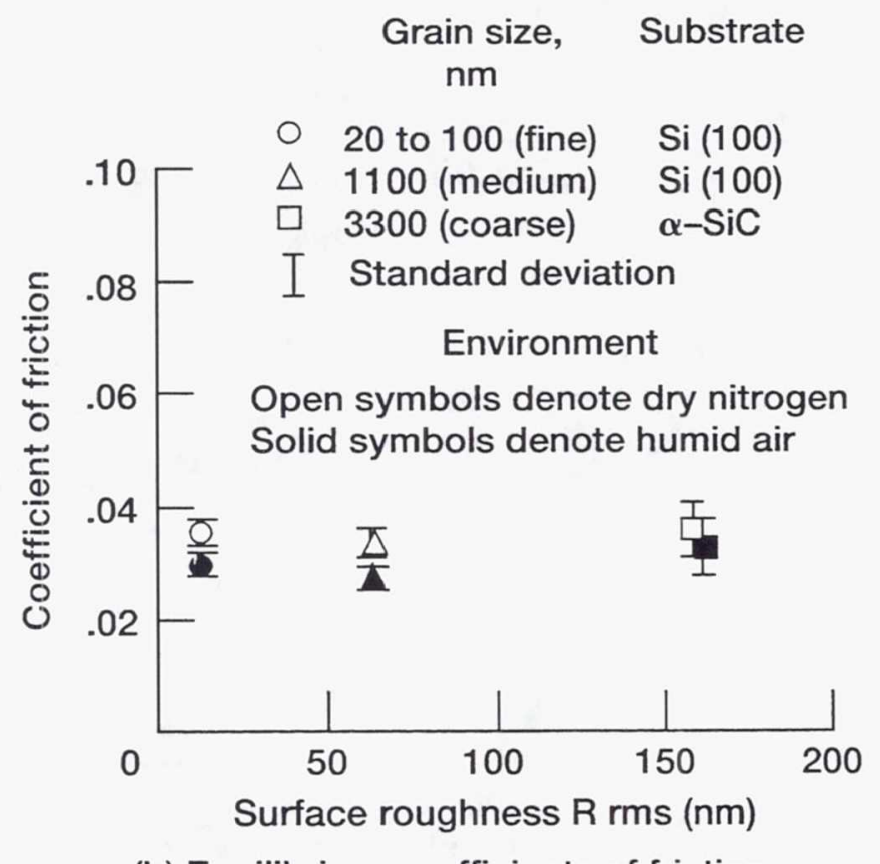

(b) Equilibrium coefficients of friction.

Figure 6. - Coefficients of friction as function of initial surface roughness of diamond films in humid air and in dry nitrogen.

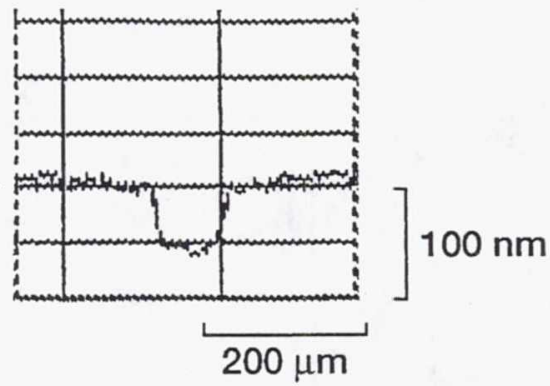

(a) Fine-grain diamond film (grain size $=20$ to $100 \mathrm{~nm}$; surface roughness $R \mathrm{rms}=15 \mathrm{~nm}$ ).

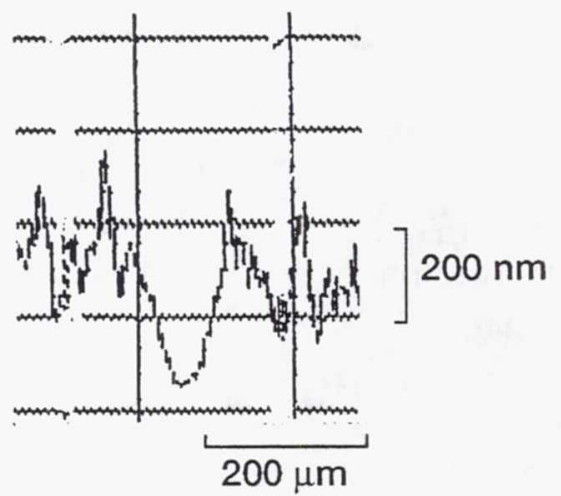

(b) Medium-grain diamond film (grain size = $1100 \mathrm{~nm}$; surface roughness $R \mathrm{rms}=63 \mathrm{~nm}$ ).

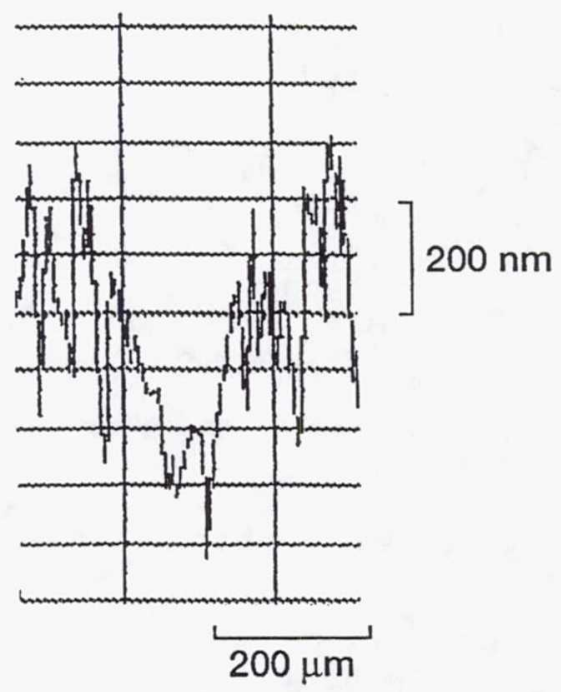

(c) Coarse-grain diamond film (grain size = $3300 \mathrm{~nm}$; surface roughness $\mathrm{R} \mathrm{rms}=160 \mathrm{~nm}$ ).

Figure 7. - Wear tracks (grooves) on diamond films after 30000 passes of bulk diamond pin in dry nitrogen. 


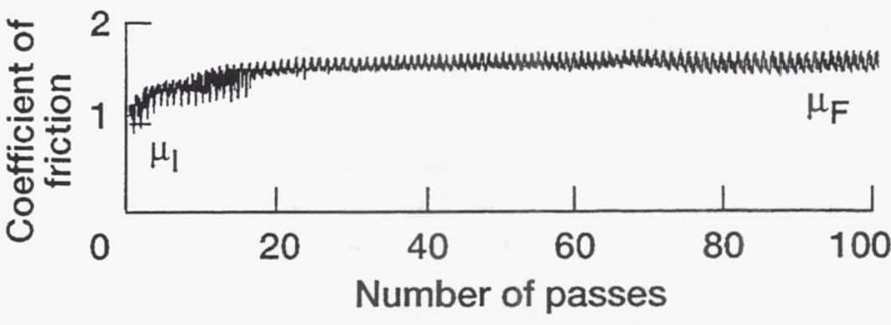

Figure 9. - Typical friction trace for bulk diamond pin in contact with diamond film deposited on $\alpha$-SiC substrate in vacuum ( $\mu_{I}=$ initial coefficient of friction; $\mu_{\mathrm{F}}=$ equilibrium coefficient of friction).

Grain size, Substrate $\mathrm{nm}$

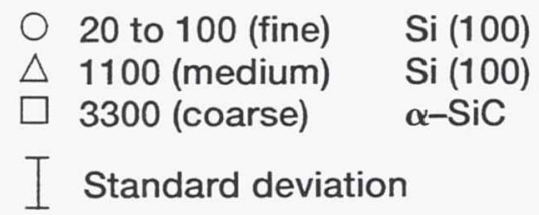

Environment

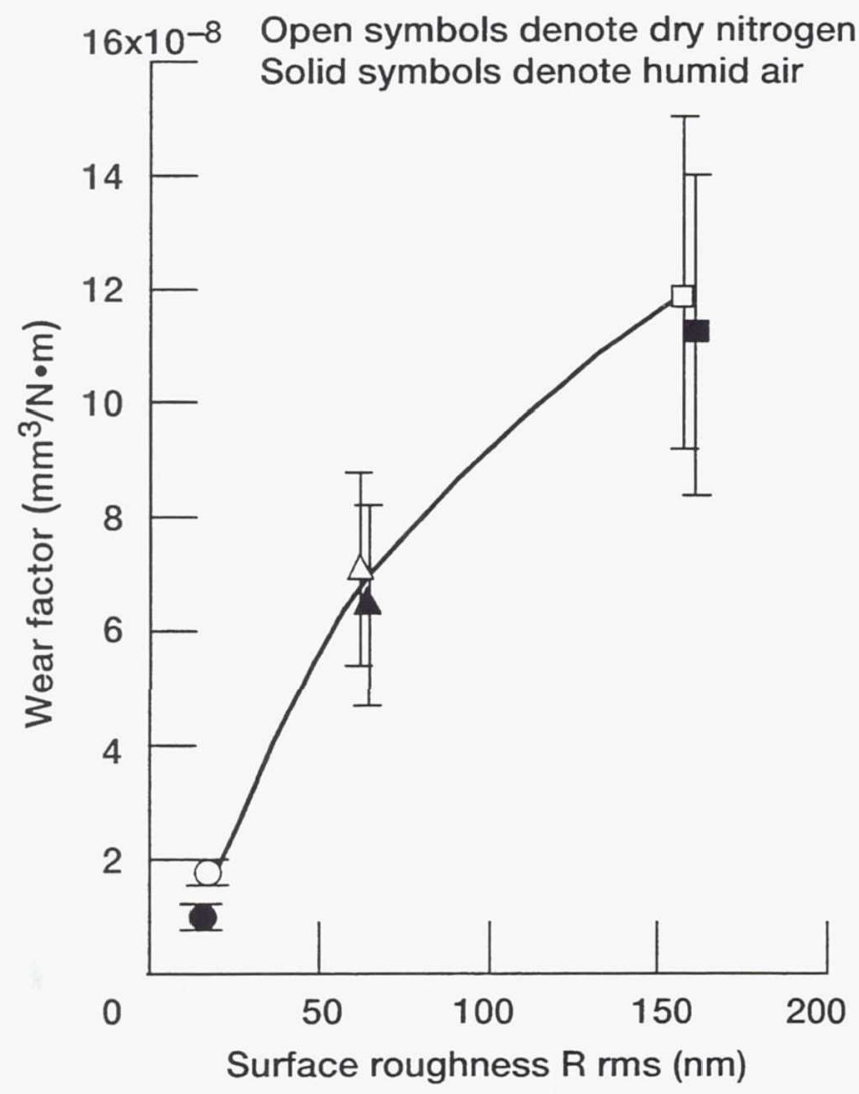

Figure 8. - Wear factors of diamond films as function of initial surface roughness in humid air and in dry nitrogen.

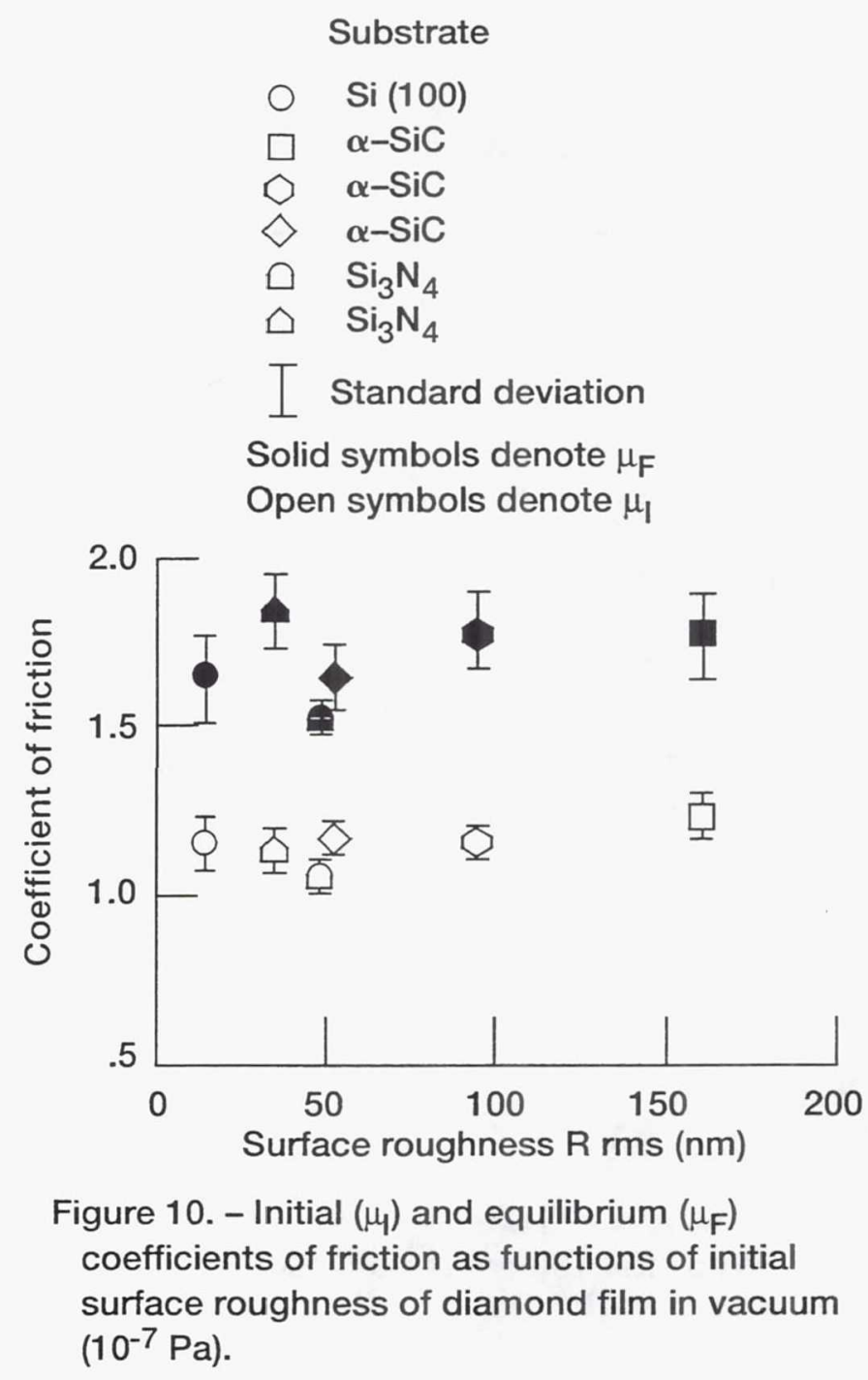




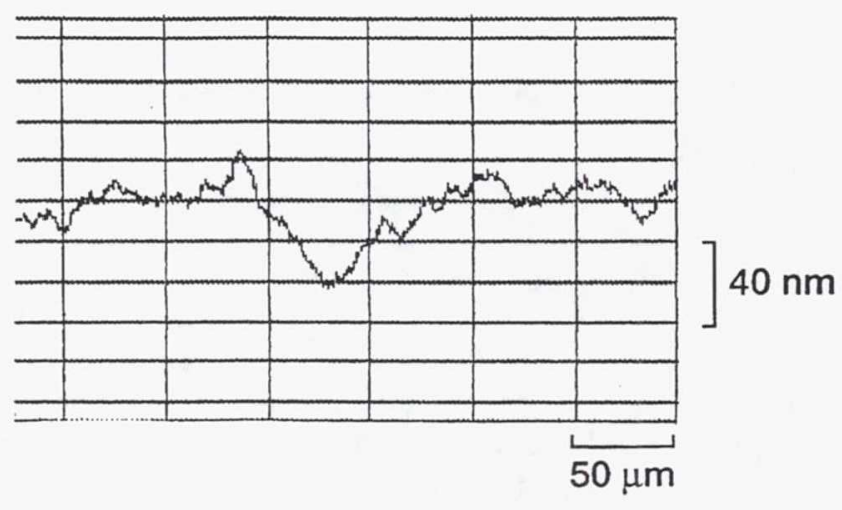

(a) Fine-grain diamond film on $\mathrm{Si}$ (grain size $=20$ to $100 \mathrm{~nm}$; surface roughness $\mathrm{R} \mathrm{rms}=15 \mathrm{~nm}$ ).

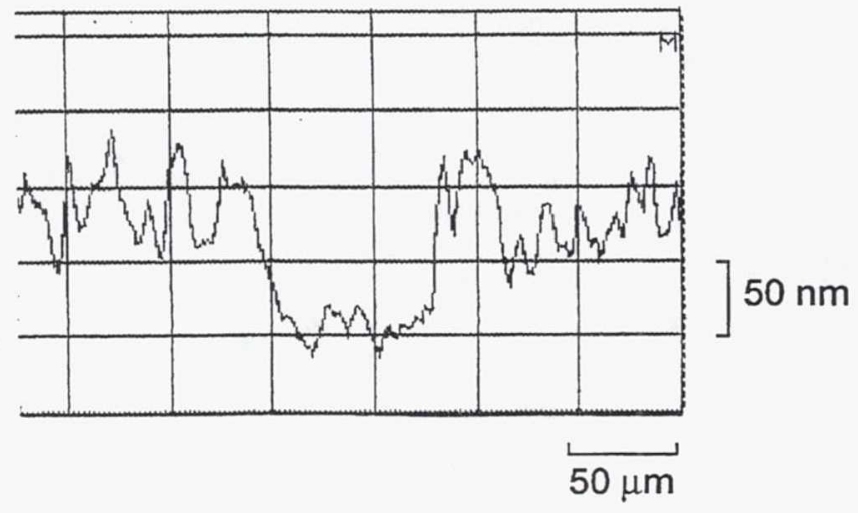

(b) Medium-grain diamond film on $\mathrm{Si}_{3} \mathrm{~N}_{4}$ (grain size $=1000 \mathrm{~nm}$; surface roughness $\mathrm{R} \mathrm{rms}=52 \mathrm{~nm}$ ).

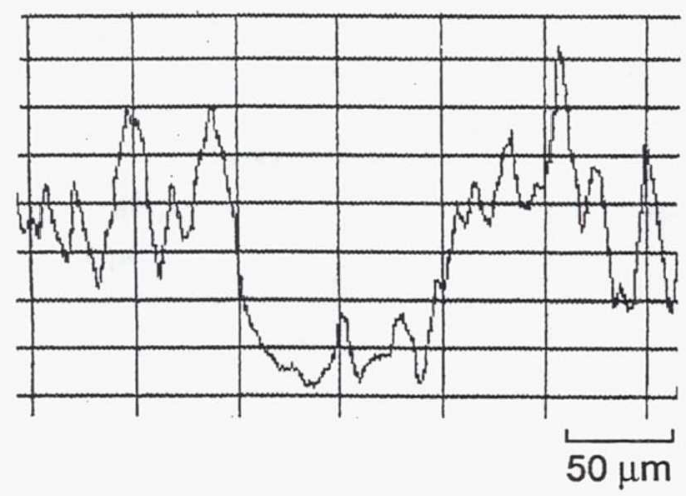

(c) Coarse-grain diamond film on $\alpha-\mathrm{SiC}$ (grain size $=1500 \mathrm{~nm}$; surface roughness R rms $=92 \mathrm{~nm}$ ).

Figure 11. - Wear tracks (grooves) on diamond films after 100 passes of bulk diamond pin in vacuum $\left(10^{-7} \mathrm{~Pa}\right)$.

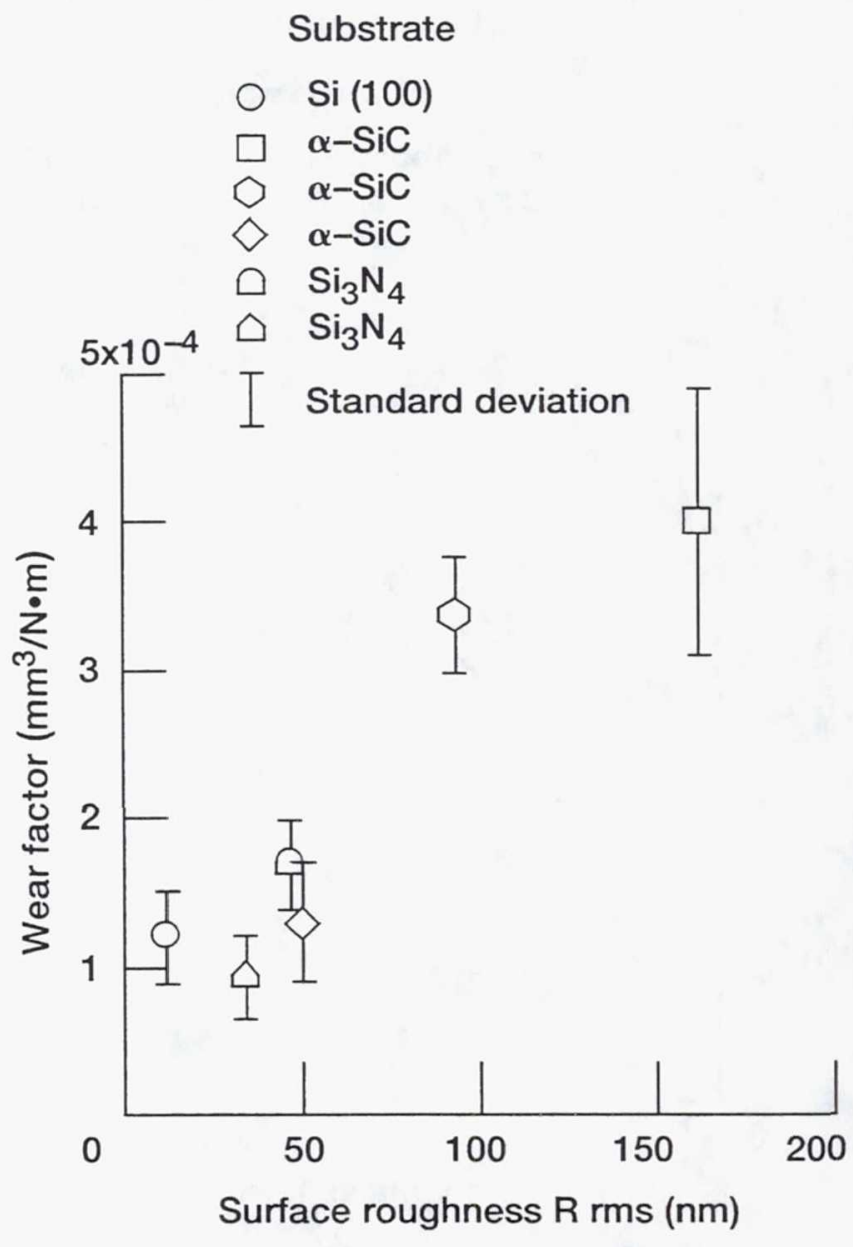

Figure 12. - Wear factors of diamond films in vacuum $\left(10^{-7} \mathrm{~Pa}\right)$ as function of initial surface roughness. 
Public reporting burden for this collection of information is estimated to average 1 hour per response, including the time for reviewing instructions, searching existing data sources, gathering and maintaining the data needed, and completing and reviewing the collection of information. Send comments regarding this burden estimate or any other aspect of this collection of information, including suggestions for reducing this burden, to Washington Headquarters Services, Directorate for information Operations and Reports, 1215 Jefferson Davis Highway, Suite 1204, Arlington, VA 22202-4302, and to the Office of Management and Budget, Paperwork Reduction Project (0704-0188), Washington, DC 20503.

\begin{tabular}{|l|c|c|}
\hline 1. AGENCY USE ONLY (Leave blank) & $\begin{array}{c}\text { 2. REPORT DATE } \\
\text { January } 1993\end{array}$ & $\begin{array}{r}\text { 3. REPORT TYPE AND DATES COVERED } \\
\text { Technical Memorandum }\end{array}$ \\
\hline
\end{tabular}

4. TITLE AND SUBTITLE

Friction and Wear of Plasma-Deposited Diamond Films

6. AUTHOR(S)

Kazuhisa Miyoshi, Richard L.C. Wu, Alan Garscadden, Paul N. Barnes, and Howard E. Jackson

\section{PERFORMING ORGANIZATION NAME(S) AND ADDRESS(ES)}

National Aeronautics and Space Administration

Lewis Research Center

Cleveland, Ohio 44135-3191

9. SPONSORING/MONITORING AGENCY NAMES(S) AND ADDRESS(ES)

National Aeronautics and Space Administration

Washington, D.C. 20546-0001
5. FUNDING NUMBERS

WU-506-43-11

8. PERFORMING ORGANIZATION REPORT NUMBER

E-7431

10. SPONSORING/MONITORING AGENCY REPORT NUMBER

NASA TM-105926

11. SUPPLEMENTARY NOTES

Prepared for the International Conference on Metallurgical Coatings and Thin Films sponsored by the American Vacuum Society, San Diego, California April 19-23, 1993. Kazuhisa Miyoshi, NASA Lewis Research Center, Cleveland, Ohio; Richard L.C. Wu, Universal Energy Systems, Inc., Dayton, Ohio 45432; Alan Garscadden and Paul N. Barnes, Wright Laboratory, Wright-Patterson Air Force Base, Ohio 45433; and Howard E. Jackson, Department of Physics, University of Cincinnati, Cincinnati, Ohio 45221. Responsible person, Kazuhisa Miyoshi, (216) $433-6078$.

12a. DISTRIBUTION/AVAILABILITY STATEMENT 12 DISTRIBUTION CODE

Unclassified - Unlimited

Subject Category 27

13. ABSTRACT (Maximum 200 words)

Reciprocating sliding friction experiments in humid air and in dry nitrogen and unidirectional sliding friction experiments in ultrahigh vacuum were conducted with a natural diamond pin in contact with microwave-plasma-deposited diamond films. Diamond films with a surface roughness (R rms) ranging from 15 to $160 \mathrm{~nm}$ were produced by microwave-plasma-assisted chemical vapor deposition. In humid air and in dry nitrogen, abrasion occurred when the diamond pin made grooves in the surfaces of diamond films, and thus the initial coefficients of friction increased with increasing initial surface roughness. The equilibrium coefficients of friction were independent of the initial surface roughness of the diamond films. In vacuum the friction for diamond films contacting a diamond pin arose primarily from adhesion between the sliding surfaces. In these cases, the initial and equilibrium coefficients of friction were independent of the initial surface roughness of the diamond films. The equilibrium coefficients of friction were 0.02 to 0.04 in humid air and in dry nitrogen, but 1.5 to 1.8 in vacuum. The wear factor of the diamond films depended on the initial surface roughness, regardless of environment; it increased with increasing initial surface roughness. The wear factors were considerably higher in vacuum than in humid air and in dry nitrogen.

14. SUBJECT TERMS

Diamond films; Friction; Wear; Vacuum

15. NUMBER OF PAGES

17. SECURITY CLASSIFICATION OF REPORT

Unclassified
18. SECURITY CLASSIFICATION OF THIS PAGE

Unclassified
19. SECURITY CLASSIFICATION OF ABSTRACT Unclassified 
National Aeronautics and

Space Administration

Lewis Research Center

Cleveland, Ohio 44135

Otticial Business

Penalty for Private Use $\$ 300$

\section{FOURTH CLASS MAIL}

ADDRESS CORRECTION REQUESTED
|||||

National Aeronautics and

Space Administration

NASA 45 ! 